\title{
Sequence, distribution and chromosomal context of class I and class II pilin genes of Neisseria meningitidis identified in whole genome sequences
}

Mirka E Wörmann ${ }^{1}$, Corey L Horien ${ }^{2}$, Julia S Bennett ${ }^{3}$, Keith A Jolley ${ }^{3}$, Martin C J Maiden ${ }^{3}$, Christoph M Tang ${ }^{1}$, Ellen $L$ Aho $^{2^{*}}$ and Rachel M Exley ${ }^{1^{*}}$

\begin{abstract}
Background: Neisseria meningitidis expresses type four pili (Tfp) which are important for colonisation and virulence. Tfp have been considered as one of the most variable structures on the bacterial surface due to high frequency gene conversion, resulting in amino acid sequence variation of the major pilin subunit (PilE). Meningococci express either a class I or a class II pilE gene and recent work has indicated that class II pilins do not undergo antigenic variation, as class II pilE genes encode conserved pilin subunits. The purpose of this work was to use whole genome sequences to further investigate the frequency and variability of the class II pilE genes in meningococcal isolate collections.

Results: We analysed over 600 publically available whole genome sequences of $\mathrm{N}$. meningitidis isolates to determine the sequence and genomic organization of pilE. We confirmed that meningococcal strains belonging to a limited number of clonal complexes (ccs, namely $\mathrm{cc1}, \mathrm{cc5}$, cc8, cc11 and cc174) harbour a class II pilE gene which is conserved in terms of sequence and chromosomal context. We also identified pils cassettes in all isolates with class II pilE, however, our analysis indicates that these do not serve as donor sequences for pilE/pils recombination. Furthermore, our work reveals that the class II pilE locus lacks the DNA sequence motifs that enable (G4) or enhance (Sma/Cla repeat) pilin antigenic variation. Finally, through analysis of pilin genes in commensal Neisseria species we found that meningococcal class II pilE genes are closely related to pilE from Neisseria lactamica and Neisseria polysaccharea, suggesting horizontal transfer among these species.

Conclusions: Class II pilins can be defined by their amino acid sequence and genomic context and are present in meningococcal isolates which have persisted and spread globally. The absence of G4 and Sma/Cla sequences adjacent to the class II pilE genes is consistent with the lack of pilin subunit variation in these isolates, although horizontal transfer may generate class II pilin diversity. This study supports the suggestion that high frequency antigenic variation of pilin is not universal in pathogenic Neisseria.
\end{abstract}

Keywords: Type four pilus, Neisseria meningitidis, Class I pilin, Class II pilin, Antigenic variation

\footnotetext{
* Correspondence: aho@cord.edu; rachel.exley@path.ox.ac.uk

2Department of Biology, Concordia College, Moorhead, MN, USA

'Sir William Dunn School of Pathology, University of Oxford, Oxford OX1 3RE, UK

Full list of author information is available at the end of the article
}

\section{Ciomed Central}

(C) 2014 Wörmann et al.; licensee BioMed Central Ltd. This is an Open Access article distributed under the terms of the Creative Commons Attribution License (http://creativecommons.org/licenses/by/2.0), which permits unrestricted use, distribution, and reproduction in any medium, provided the original work is properly credited. The Creative Commons Public Domain Dedication waiver (http://creativecommons.org/publicdomain/zero/1.0/) applies to the data made available in this article, unless otherwise stated. 


\section{Background}

Neisseria meningitidis, the meningococcus, is an important human pathogen, being a leading cause of septicaemia and bacterial meningitis. Systemic disease has a high case fatality rate and can progress rapidly over the course of a few hours [1]. Different epidemiological patterns of meningococcal disease are observed across the world and Multi-Locus Sequence Typing (MLST) has been instrumental in characterising strains and identifying major pathogenic lineages (clonal complexes) responsible for disease [2]. Many $N$. meningitidis isolates from invasive disease belong to 'hyperinvasive lineages' [3], such as clonal complexes (cc)- 5, cc11, cc269, cc32 and cc41/44. Whole genome sequence (WGS) analyses have recently been carried out on large isolate collections, including those used to develop MLST [2,4]. These WGS data represent a valuable resource for investigating the frequency and variability of genes encoding meningococcal virulence traits.

Type four pili (Tfp) are important virulence factors for $N$. meningitidis, contributing to colonisation and disease [5]. Adhesion of meningococci to epithelial and endothelial cells is mediated by Tfp interacting with host cells, promoting bacterial aggregation and mediating microcolony formation [6]. Neisseria Tfp are also essential for the acquisition of exogenous DNA and emergence of strains with novel, heritable characteristics [7]. Along with LPS, porins and the polysaccharide capsule, Tfp have been considered as key meningococcal surface structures which undergo extensive variation. Studies on the Tfp of the related pathogen Neisseria gonorrhoeae have revealed that changes in the amino acid sequence of PilE (pilin), the major subunit of Tfp, arise through introduction of segments of non-expressed pilS cassettes into the pilE expression locus by a unidirectional DNA recombination event known as gene conversion $[8,9]$. This process is enhanced by the presence of the Sma/ Cla repeat and requires the guanine quartet (G4) adjacent to the pilE expression locus. The Sma/Cla repeat is a 66 bp element present at the $3^{\prime}$ end of pilE that is proposed to bind proteins to facilitate recombination [10]. The G4 sequence is located upstream of pilE and is necessary for pilin conversion, putatively by acting as a recombination initiation structure $[11,12]$. More recently a cis-acting small RNA has been proposed to assist in the melting of the DNA duplex to allow formation of the G4 structure [13]. High frequency antigenic variation generates remarkable PilE diversity, with most of the sequence changes localised to the regions of the protein that are exposed on the surface of the pilus fibre [14], most notably within a region (D-region) located near the Cterminus of the protein. Gonococcal pilin variation has been detected both in vitro [15] and in human volunteer studies [16]. Meningococcal pilin variation has also been shown in a limited number of isolates in vitro [17] and in vivo $[18,19]$ and has been proposed to influence Tfpmediated adhesion [20-22], serum resistance [23] and to serve as a mechanism for immune evasion.

Two classes of Tfp (class I and class II) have been described in the Neisseria, based on the cross reaction of PilE with a monoclonal antibody, SM1 [24]. This antibody recognizes a conserved epitope in class I, but not class II pilin [25]. Furthermore, class II pilins lack most of the Dregion typically seen in gonococccal and meningococcal class I pilins [26]. The class II pilE gene was initially described in the $N$. meningitidis serogroup C strain, FAM18 and subsequently found in non-pathogenic species, $N$. lactamica and $N$. cinerea [27]. Until recently, all pathogenic Neisseria were thought to have the ability to alter the sequence of the major pilin subunit through gene conversion; however, pilin variation was not detected in vitro in N. meningitidis strain FAM18, or strain NMB, which both express class II pilin [17,28]. Furthermore, in a previous study we found that cc8 and cc11 isolates of $N$. meningitidis collected from the UK over a period spanning 30 years harboured highly conserved class II pilE genes which lack variation characteristic of gene conversion [29]. Similar analysis of the sequence of pilE genes in meningococcal isolates from China demonstrated that strains in $\mathrm{cc} 1$ and cc5 also have a highly conserved class II pilE gene [30].

These findings suggest that high frequency pilE gene conversion is not universal in pathogenic Neisseria, an observation which has important implications for understanding the role of Tfp variation during colonisation, transmission and disease. Therefore, in this study we interrogated genome sequences of over 600 meningococcal isolates to further examine the distribution and sequence conservation of class II pilE genes, and to identify differences in the class I and class II pilE loci which might explain the lack of variability of the class II pilin gene. Our analyses showed that the class I and class II pilE genes are in distinct chromosomal contexts and that isolates with class II pilE contain a more limited repertoire of pilS cassettes. Furthermore, the G4 and Sma/Cla sequences, which are important for pilin variation, are absent from the class II pilE locus. Finally we present evidence of horizontal transfer of class II pilE genes between pathogenic and non-pathogenic Neisseria species, which has implications for the evolution of these bacteria.

\section{Results}

Distribution and diversity of class I and class II pilE in N. meningitidis

Draft whole genome sequences hosted on the PubMLST website [4] were interrogated to determine the distribution and diversity of class II pilE genes among $N$. meningitidis isolates. This included the Meningitis Research Foundation Meningococcus Genome Library (MRF-MGL), which 
contains the sequences of 514 disease-associated isolates from patients in England, Wales and Northern Ireland during a single epidemiological year (2010 to 2011), and which represents recently circulating UK disease causing meningococci. In addition WGS of the 107 strains of $N$. meningitidis used to develop and validate MLST, which comprises a diverse collection of meningococci isolated from 37 countries collected over a 59 year period (1937 to 1996) [2] were examined.

Pilin genes in this collection of isolates were identified by online BLAST analyses using full-length or partial class I or class II pilE sequences. Homologous sequences were manually extracted, translated and designated as either class I or class II pilE based upon relatedness to known pilin genes and the presence of the SM1 motif (EYYLN) in the translated sequence. It was not possible to identify full-length pilE sequences in all WGS, most likely due to incomplete assembly. From the MRF genomes a total of 201 full-length class I pilE coding sequences and 31 class II pilE coding sequences were identified and in the MLST collection of 107 genomes, 12 full-length class I pilE coding sequences and 44 class II pilE coding sequences were found (a breakdown of pilE analysis is described in Additional file 1). Therefore, in total 213 isolates with a full-length class I pilE gene and 75 isolates with a full-length class II pilE gene were identified (listed in Additional files 2 and 3). No isolates that contained both a class I and a class II pilE gene were identified.

While there was no association of class II pilE with serogroup, country or year, the presence of a class II pilE gene was consistently associated with a limited group of clonal complexes i.e. cc1, cc5, cc8, cc11 and cc174; no WGS data from isolates belonging to these ccs contained a class I pilE gene. Only cc4 contained isolates with either class I pilE (ID 35 and ID 613) or class II pilE (ID 1) (see Additional files 2 and 3). Thus, the data from the 107 MLST and MRF isolate collections confirmed that the presence of class II pilin is a characteristic of a distinct subset of meningococcal lineages.

The extent of sequence diversity among the 213 class I and the 75 class II pilE sequences identified in this work was examined, including pilE sequences from eight available reference genome sequences, providing an additional six class I pilE genes and two class II pilE sequences (Additional file 4). Allele numbers were assigned to the full-length pilE (NEIS0210) coding sequences. Each of the 219 class I pilE genes was a unique allele (Table 1), even in strains of the same sequence type, isolated in the same country and year. In contrast, only 15 class II pilE alleles were identified from 77 meningococci isolated from 29 countries over 74 years, highlighting the sequence conservation of class II pilE genes (Table 1). There was also evidence for an association between certain class II pilE
Table 1 Analysis of class I and class II pilE sequence diversity

\begin{tabular}{llllll}
\hline pilE & No. isolates & No. countries & Years & No. ccs & No. alleles \\
\hline Class I & 219 & 5 & $1963-2011$ & $21+n / a$ & 219 \\
Class II & 77 & 29 & $1937-2011$ & 6 & 15 \\
\hline
\end{tabular}

n/a: not assigned to clonal complex.

alleles and particular clonal complexes (Figure 1). For example, $90 \%$ (28/31) of cc11 isolates harboured an identical pilE (allele 4). Likewise, all 13 cc5 isolates harboured the same pilE coding sequence (allele 15). This allele was also found in strains belonging to $\mathrm{cc} 1$ and $\mathrm{cc} 174$, suggesting horizontal transfer of class II pilE. Further evidence of this was allele 14, which was present in strains belonging to cc1 and cc8.

Analysis of the predicted protein sequences showed that for class I pilins most variation occurred within the C-terminal D-region (Figure 2A), as expected based on previous studies $[8,14,31]$. The 219 different class I pilE alleles encode 219 different pilin subunits with 194 different D-regions (not shown). In contrast, alignment of the 15 unique class II pilins we identified demonstrates that variation occurs over the entire $\mathrm{C}$-terminal domain and only six different D-regions are encoded (Figure 2B).

\section{Analysis of pilE/pilS loci in isolates with class I or class II pilE}

In the class I pilin-producing meningococci with closed genome sequences (MC58 [32] and Z2491 [33]) the class I pilE gene and eight pilS cassettes are found in a single locus, while in the closed genomes of class II pilinproducing meningococci FAM18 [34] and WUE2594 [35], this locus contains a reduced number of pilS cassettes and class II pilE is located at a distinct locus (Figure 3). The class I pilE gene is located between $f k b p$ (which encodes a putative peptidyl-prolyl cis-trans isomerase) and lpxC (UDP-3-O-[3-hydroxymyristoyl] $\mathrm{N}$-acetylglucosamine deacetylase) (Figure 3A). The silent pilS cassettes precede pilE and, although there are eight cassettes in each instance, there is variation in their length and spacing. In contrast the class II pilE gene is situated between catalase $(k a t A)$ and a hypothetical protein $(h p)$, with a conserved hypothetical (chp) and putative oligopeptidase A gene ( $\mathrm{prlC}$ ) further downstream (Figure 3B). The catalase region in the genomes of MC58 and Z2491 does not contain a class II pilE gene (Figure 3A). Finally, in FAM18 and WUE2594, two or three pils cassettes respectively are located in the region between $f k b p$ and $l p x C$ (Figure 3B).

To assess the chromosomal context of class I and class II pilE genes and their associated pilS repertoires in different groups of meningococcal isolates, we annotated the $f k b p-l p x C$ region and the $k a t A-p r l C$ regions in a subset of WGS in the PubMLST database. Where possible groups of isolates with the same sequence type or in the 


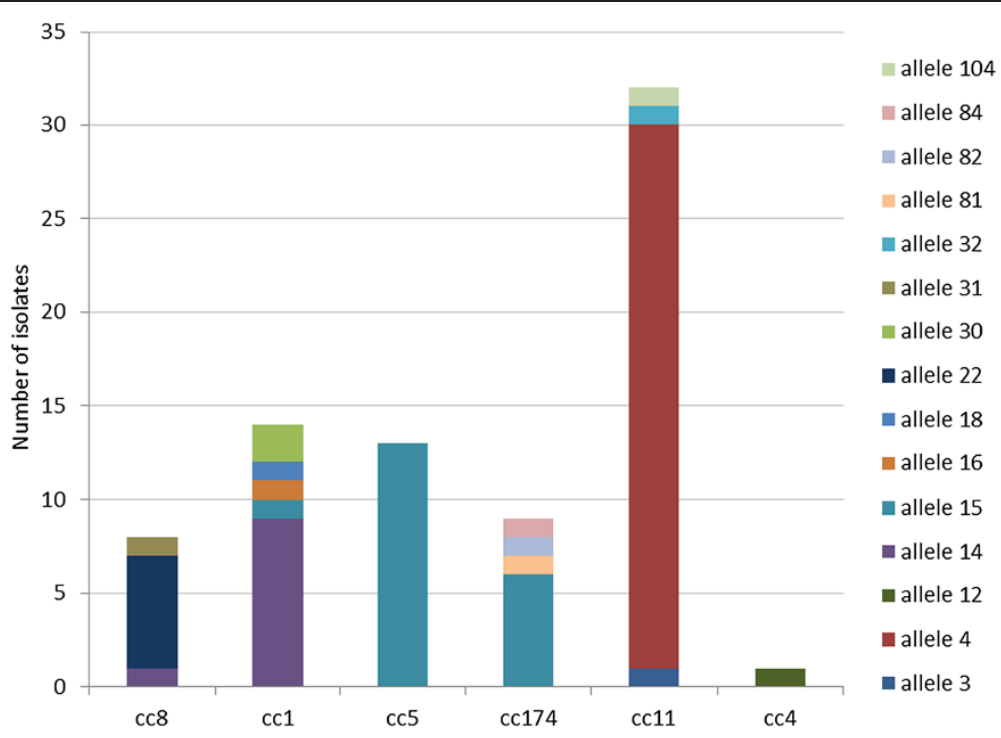

Figure 1 Distribution of class II pilE nucleotide alleles among clonal complexes. A total of 15 different alleles were identified from 77 meningococcal isolates. Isolates from cc1 displayed the most diversity, with five unique pilE sequences found in 14 isolates. All 13 cc5 strains had identical pilE sequence. Alleles 14 and 15 were found in isolates from 2 or 3 different clonal complexes respectively. Clonal complex and pilE alleles are indicated.

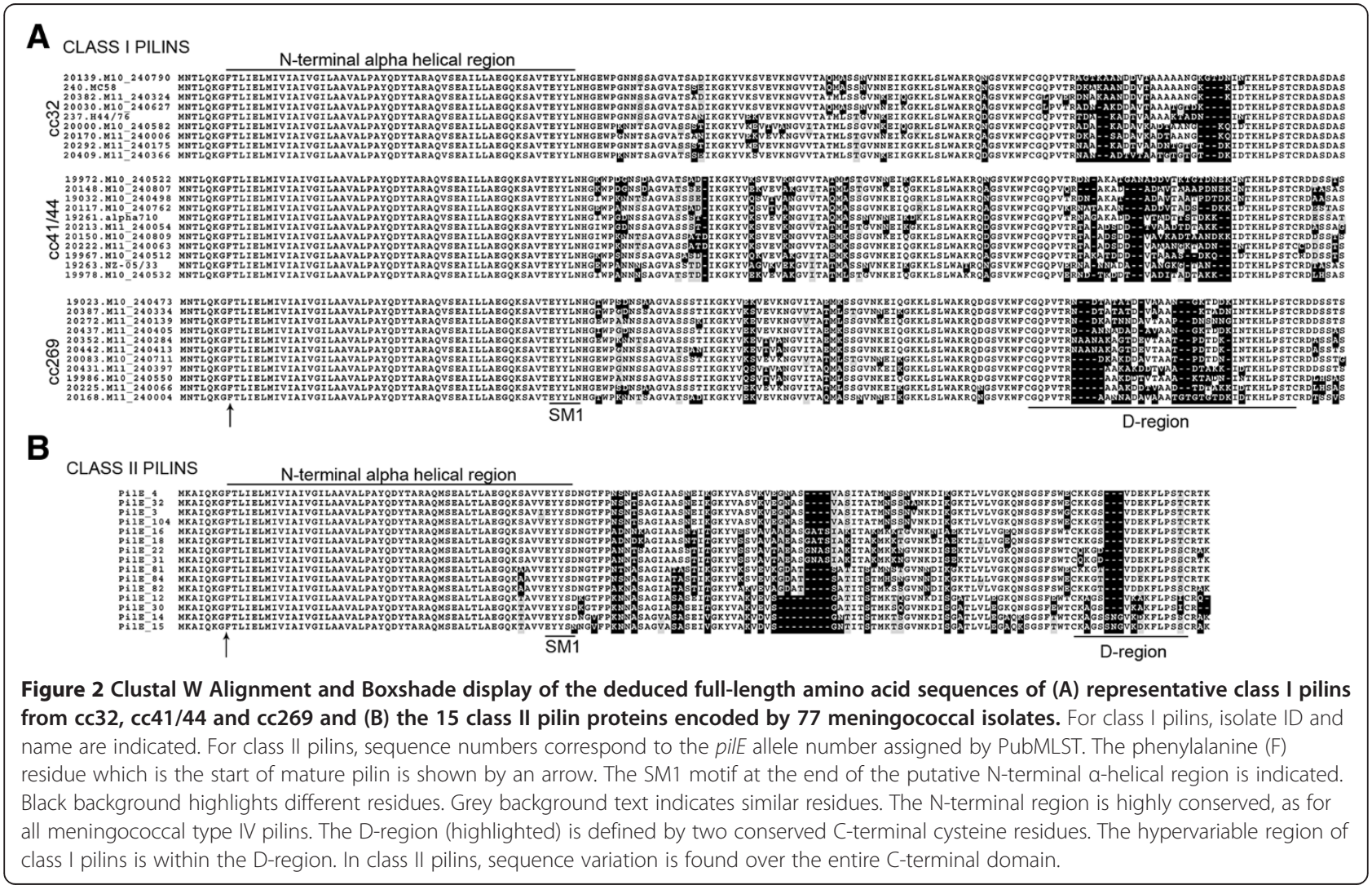




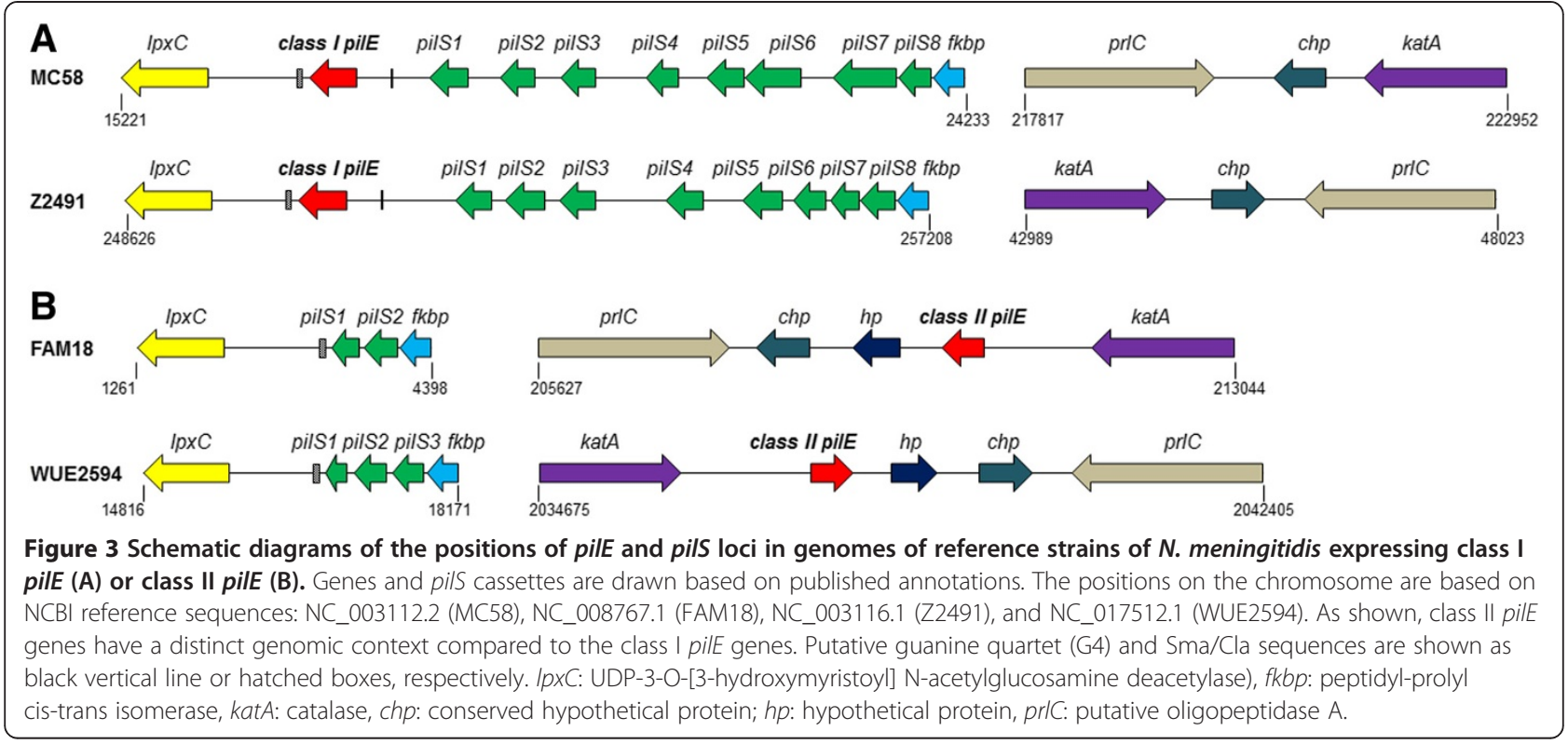

same clonal complex were examined, to enable comparisons both within and between groups of related meningococci. The pils cassettes were defined on the basis of their homology to pile coding sequences. In some cases the lack of available contiguous sequence prevented identification of adjacent open reading frames; examples of class I and class II pilE/pilS regions from genomes where the assembly was sufficient are shown in Figure 4 and Additional files 5 and 6.

Class I pilE genes were found adjacent to pilS cassettes and between $f k b p$ and $l p x C$ in 29 of the 34 annotated genomes. In the remaining five genomes, it was not possible to determine whether pilE was adjacent to $l p x C$ (Isolate IDs 20170 and 20382, Figure 4) or pilE genes encoding atypical pilin variants (with an extended and truncated C-terminus, respectively) were found, but these were not within in the $f k b p$-lpxC locus (cc32 isolates ID 20161 and ID 20250, Figure 4A). Likewise in cc269 isolate M10_240550 (ID 19986), the pilE/S locus was interrupted by a region homologous to the $t s p B$ region of strain FAM18 (see Additional file 5). None of these isolates harboured a pilE gene in the katA-prlC region (Figure 4B).

Analysis of pilS cassettes in the $f k b p-l p x C$ region of the class I pilE-containing isolates revealed diversity in the spacing, number and sequence of the silent cassettes, both among clonal complexes and among isolates belonging to the same clonal complex (see Table 2 and schematic loci in Figure 4 and Additional file 5). This diversity was particularly evident in cc41/44, where we identified 52 different pilS sequences from a total of 84 cassettes from 12 genomes. It should be noted that the analysis of class I strains was specifically targeted to annotate pilS cassettes within the $f k b p-l p x C$ region and the presence of additional pilS cassettes elsewhere in these genomes could not be excluded. Therefore this analysis represents the minimum diversity of pilS in isolates with class I pilE.

The pilS and pilE sequences within each isolate were examined for evidence of past intra-strain gene conversion events. Of note, for all isolates with a class I pilE gene, it was possible to identify pilS cassettes with sequences corresponding to those found in the PilE Dregion. In most cases the D-region was a hybrid, likely resulting from multiple recombination events, whilst in some instances a single pils donor could be proposed based on full sequence identity (not shown).

Annotation of genomes with class II pilE revealed that this gene was present in a region defined as the katA$\mathrm{prlC}$ region (annotated regions are shown in Figure $4 \mathrm{C}$ and $\mathrm{D}$, and Additional file 6). All these regions had identical genetic organisation, except isolate 7891 (ID7, Finland, 1975), which lacks the conserved hypothetical protein (chp, NMC0207 in FAM18). Furthermore, all genomes with class II pilE had fewer pils cassettes than class I isolates - usually two or three in the region between $f k b p$ and $l p x C$ (Figure $4 C$ and Table 2). Further BLAST analyses of these class II genomes were performed to identify whether pilS cassettes were also in other loci. The only homologous sequences identified were located on contigs containing the region between $f k b p$ and $l p x C$ (not shown), suggesting that there was only a single pils locus in these isolates. Although incomplete genome assembly preventing additional pilS identification in this study cannot be excluded, the fully assembled, closed genome sequences of reference strains FAM18 and WUE2594 do not contain pils cassettes located elsewhere. 


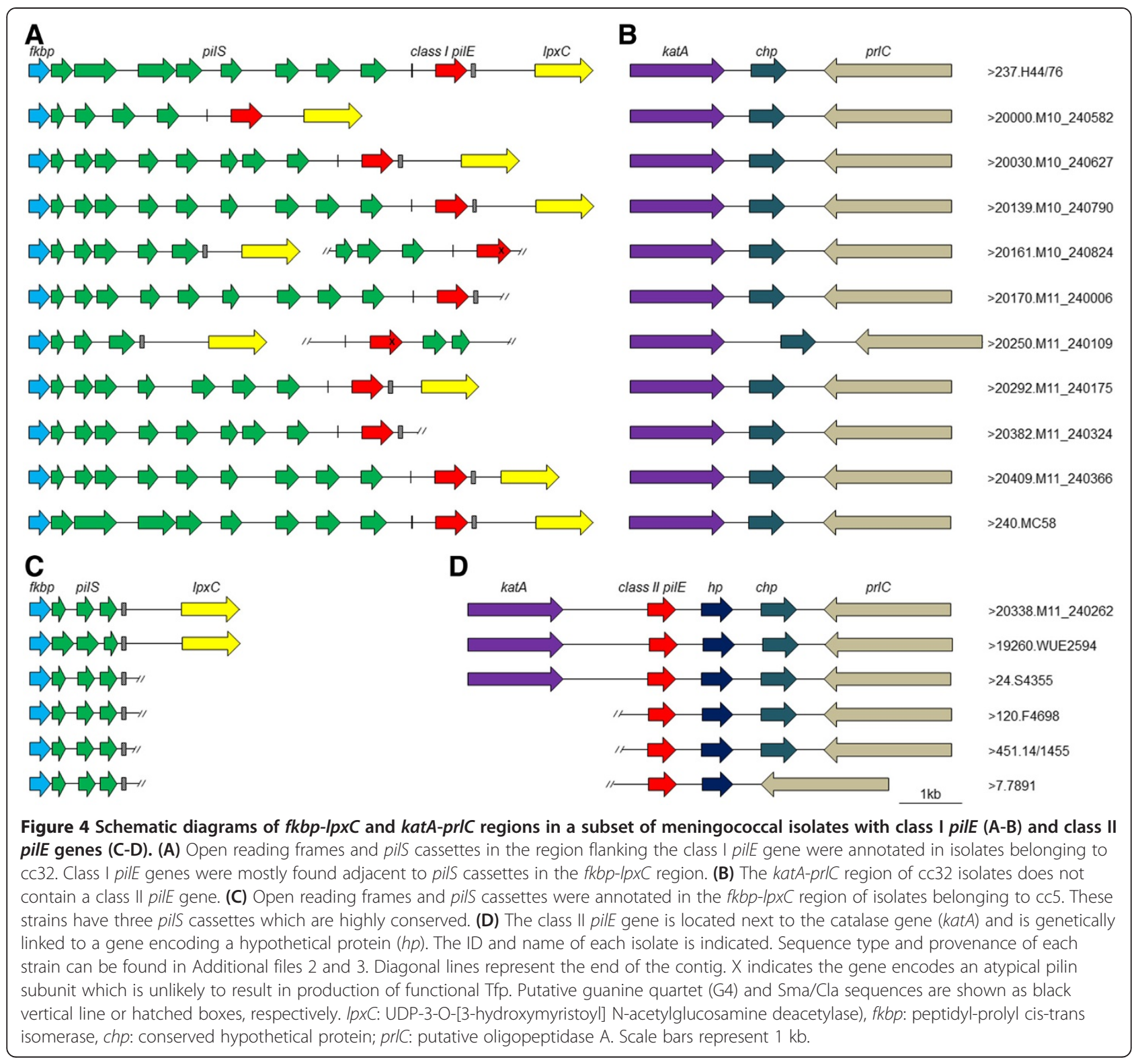

In comparison to strains with class I pilE, the pilS regions in these class II isolates were highly conserved within a clonal complex in terms of number and arrangement of pils cassettes (see Figure 4 and Additional file 6). For example, there were only four different pilS sequences out of 64 cassettes in 32 cc11 isolates (Table 2). The pilS cassettes in class II isolates shared greater similarities with class I pilE than class II pilE as previously observed [36]. Furthermore, we were unable to identify any pilS cassettes encoding sequences corresponding to those found in the D-regions of any class II pilin proteins. This finding is consistent with the hypothesis that, in these class II isolates, pils cassettes are not functioning as donors for intra-strain recombination events associated with pilin antigenic variation.

\section{Analysis of DNA sequences associated with pilE gene conversion}

The G4 and Sma/Cla elements have been shown to be required for efficient antigenic variation of pilin $[12,37]$. Each of these sequences was found in the region $5^{\prime}$ or $3^{\prime}$ to the class I pilE gene, respectively (Additional file 7). Four isolates lacked a full length Sma/Cla adjacent to pilE, three of which also presented atypical pilE sequence and/or arrangement; for example, cc41/44 isolate (ID19266) (Additional file 5), has an atypical pilE sequence and lacks a Sma/Cla sequence, while cc32 isolates ID 20161 and ID 20250 (Figure 4) have a Sma/Cla sequence adjacent to pils but in these genomes pilE is no longer found in the $f k b p$-lpxC region. 
Table 2 pilS sequence diversity in isolates with class I and class II pilE ${ }^{1}$

\begin{tabular}{llllll}
\hline $\begin{array}{l}\text { Clonal } \\
\text { complex }\end{array}$ & $\begin{array}{l}\text { pilE } \\
\text { gene }\end{array}$ & $\begin{array}{l}\text { Number of } \\
\text { isolates }\end{array}$ & $\begin{array}{l}\text { pilS } \\
\text { cassettes } \\
\text { per isolate }\end{array}$ & $\begin{array}{l}\text { Total } \\
\text { number } \\
\text { of pils } \\
\text { cassettes }\end{array}$ & $\begin{array}{l}\text { Total } \\
\text { number } \\
\text { of pils } \\
\text { alleles }\end{array}$ \\
\hline cc1 & class II & 5 & $1-3$ & 13 & 8 \\
cc5 & class II & 6 & 3 & 18 & 8 \\
cc8 & class II & 8 & 3 & 24 & 4 \\
cc11 & class II & 32 & 2 & 64 & 4 \\
cc174 & class II & 9 & $2-4$ & 27 & 6 \\
cc269 & class I & 10 & $5-9$ & 82 & 19 \\
cc41/44 & class I & 12 & $5-11$ & 84 & 52 \\
cc32 & class I & 9 & $4-9$ & 70 & 26 \\
\hline
\end{tabular}

${ }^{1}$ Isolates and pilS cassettes included in this analysis are shown in Figure 4, Additional file 5, and Additional file 6. Isolates ID 19986 (cc269), ID 20161 (cc32), and ID 20250 (cc32) were excluded due to incomplete contig assembly in regions containing pilE/pilS loci.

We also analysed the regions flanking the class II pilE alleles for presence of the $\mathrm{Sma} / \mathrm{Cla}$ and G4 sequences. We did not detect sequences corresponding to a functional G4 motif (5'-GGGTGGGTTGGGTGGG-3') [12] in the region $5^{\prime}$ to any class II pilE gene. Furthermore, the Sma/Cla repeat was absent from the region 3' to the class II pilE genes, although sequences identical or highly homologous to the published meningococcal Sma/Cla repeat [37] were present in the pils loci of these isolates (see Figure 4 and Additional files 6 and 7). Therefore, the absence of motifs that are important for pilE/pilS recombination adjacent to the class II pilE gene is likely to contribute to the lack of antigenic variation of class II pilE.

\section{Defining meningococcal class II pilins}

Class II pilins were initially identified by their lack of cross reaction with monoclonal antibody SM1 which was developed to differentiate between structural classes of Tfp on gonococci and meningococci [24]. SM1 recognises an epitope (EYYLN) present at the end of the conserved N-terminus of class I pilin [25]. Our analysis confirms that all the pilins encoded by the class I pilE contain this motif, while in the class II pilins this motif is either EYYSN (allele 15, present in 20 isolates) or EYYSD (present in all remaining isolates) (Figure 2). Western blot analysis of whole cell extracts from strains expressing class II pilin subunits with different SM1 sequences was undertaken (Figure 5). As expected, SM1 detected class I pilin in the control strain 8013 (ID 1038, allele 6, EYYLN motif) and did not recognise the class II pilin from FAM18 (ID 698, allele 3, EYYSD motif). However, class II pilin from cc5 strain 92001 (ID 597, allele 15, EYYSN motif), does cross react with SM1. This suggests that SM1 reactivity may not be sufficient to

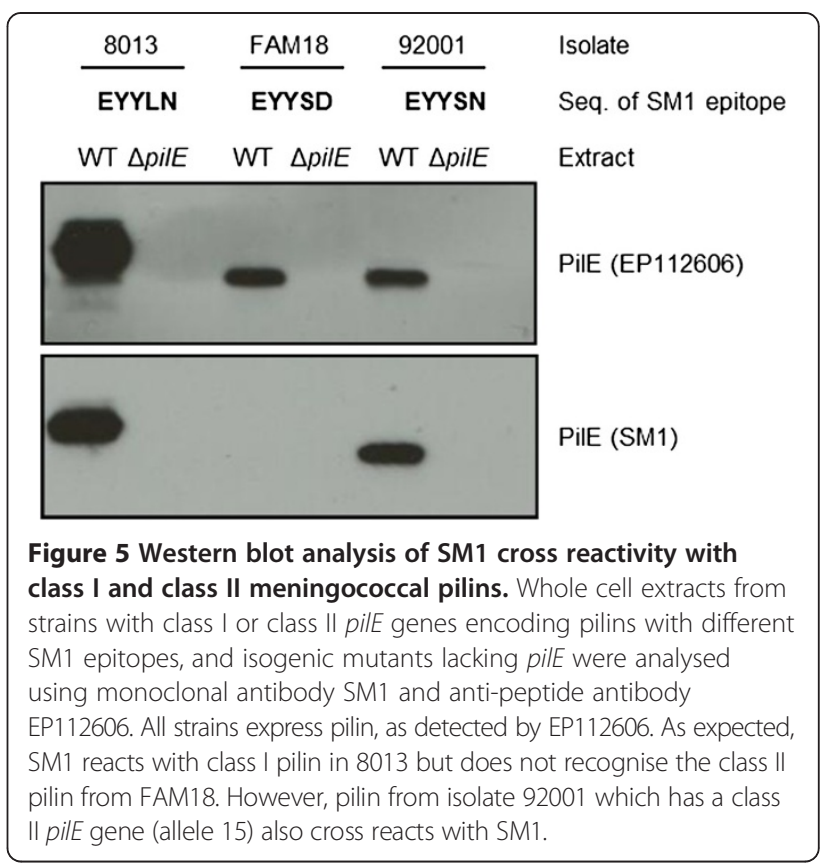

distinguish class I and class II pilin in all meningococci. We therefore compared all the pilin sequences from our analysis to examine the presence and conservation of additional differentiating features (Table 3). The sequence of the leader peptides were distinct (MNTLQKG and MKAIQKG for class I and II pilins respectively) as was the length of the C-terminus (after the second conserved cysteine), which comprises one to three amino acids in class II pilins, in contrast to seven in full-length, functional class I pilins. Most notably, all pilins encoded by the class II pilE genes were smaller, mostly due to a significantly shorter D-region, which is 13 or 16 amino acids in length compared with 28 to 40 amino acids in class I pilins (see Figure 2 and Table 3). Based on this analysis, we propose the adoption of a sequence-based distinction between class I and class II pilins, rather than a definition based on antigenic properties (reactivity with SM1).

\section{Analysis of pilE in non-pathogenic Neisseria species}

It has previously been shown that class II pilE is also present in non-pathogenic Neisseria [27,36,38]. We therefore analysed pilE sequences in genomes of 46 isolates of

Table 3 Defining features of meningococcal class I and class II type IV pilins

\begin{tabular}{lllll}
\hline Pilin & Length (AA) & Leader sequence & SM1 motif & $\begin{array}{l}\text { Length of } \\
\text { D-region }\end{array}$ \\
\hline Class I & $164-172$ & MNTLQKG & EYYLN & $28-40 \mathrm{AA}$ \\
Class II & $143-152$ & MKAIQKG & EYYSD & 13 or 16 AA \\
& & & EYYSN & \\
\end{tabular}

${ }^{1}$ Defined as the amino acids between the two C-terminal cysteine residues. 
non-pathogenic Neisseria available in pubMLST. This collection comprised 14 different species isolated between 1962 and 2003 from various hosts and sites. In total 44/46 WGS from non-pathogenic Neisseria were found to have full-length sequences homologous to pilE (These isolates are listed in Additional file 8). Split decomposition network analysis of these 44 pilE sequences with both class I and class II pilE genes from 14 N. meningitidis isolates from different clonal complexes, revealed that all meningococcal class I pilE clustered together (Figure 6). In contrast, the meningococcal class II pilE clustered on a separate branch together with pile from $N$. lactamica, $N$. polysaccharea and one $N$. cinerea isolate. Other nonpathogenic Neisseria species displayed more divergent pilE sequences. We analysed the sequences flanking the putative pilE genes in the genomes of all non-pathogenic Neisseria strains where there was sufficient sequence available on the pilE-containing contig. Nine species contained a second putative pilin gene adjacent to the initial pilE homologue (see Additional file 8), in agreement with previous reports [36,38] and in these species the regions flanking pilE did not resemble those found adjacent to either the class I or class II meningococcal pilE. In contrast, the genetic organisation of open reading frames around the pilE gene in $N$. lactamica and $N$. polysaccharea was similar to the genomic region surrounding the meningococcal class II pilE gene (annotated pilE regions of $N$. lactamica and N. polysaccharea are shown in Additional file 9), although only one non-pathogenic Neisseria isolate ( $N$. polysaccharea, isolate CCUG 27182) was found to harbour the $h p$ gene which is found in the meningococcal class II pilE locus.

\section{Discussion}

Two different classes of pilin (class I and class II) have been described in pathogenic Neisseria [24] and the genes encoding these pilins are distinguishable $[29,39]$. Here we have taken advantage of available whole genome sequences to provide a more comprehensive view of the distribution and genetic context of meningococcal class I and

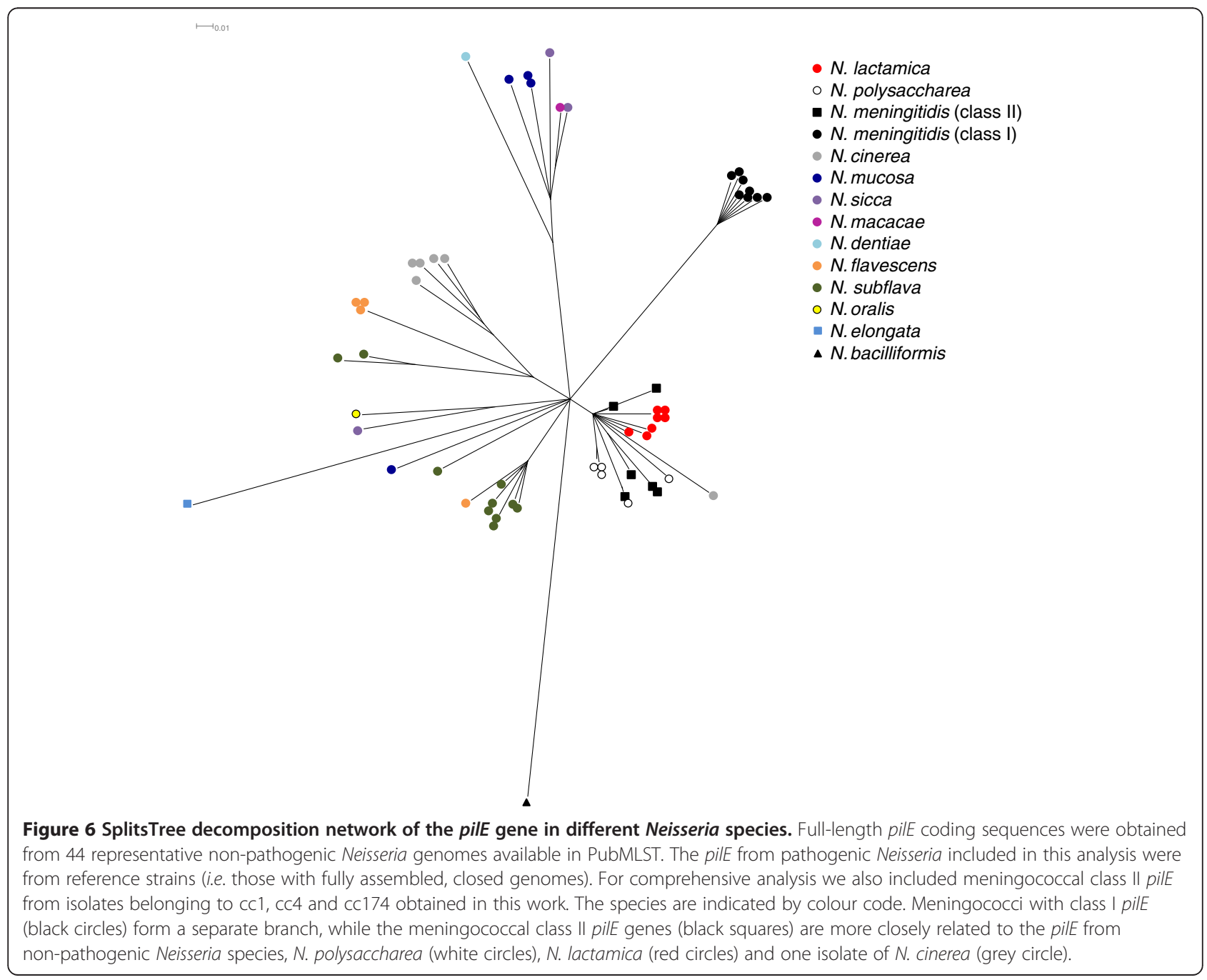


class II pilE. These analyses reveal a possible basis for the evident lack of gene conversion of class II pilE and we provide insights into the likely origin of the meningococcal class II pilE gene.

Previous analysis of pilE sequences in $N$. meningitidis isolate collections from the UK [29] and China [30] suggested an association of the class II pilE gene with certain lineages, especially those responsible for epidemic meningococcal disease (i.e. cc1, cc5 and cc11). In the present analysis, two large and well characterised isolate collections enabled the assessment of class II pilE distribution in isolates from a single country in one year, and in isolates collected over decades from diverse global locations. The data demonstrate that the association of class II pilE with these hyperinvasive lineages is maintained, regardless of the country or the year they were isolated, and highlights that class II pilE is present in lineages which are responsible for a large proportion of disease. For example, the 107 isolate MLST collection analysed in this study contains cc1 and cc5 isolates from countries of the African meningitis belt (ID 128 and ID 34, Additional file 3). The majority of epidemic meningococcal disease has occurred in this region and has been mostly caused by serogroup A meningococci belonging to cc1 or cc5 [40,41]; in particular three clones (ST-5, ST-7 and ST-2859, which are all part of cc5) have been largely responsible for meningococcal disease in Africa over the last 20 years [42-44].

In addition, we found class II pilE genes in strains from cc174 and cc4. An increase in carriage of cc174 strains was reported in the UK from 1999-2001 [45] and isolates from cc174 accounted for approximately $20 \%$ of serogroup Y meningococcal disease during 2007-2009 in the UK and were associated with disease in older age groups [46]; however, only 9/514 disease isolates from the UK in 2010-2011 belong to this complex. Therefore, there is currently insufficient information to determine whether strains belonging to this clonal complex are hyperinvasive and/or associated with epidemic disease. It is possible that the groups of strains with class II pilE may expand with increasing availability of strain collections and whole genome sequences and consequently the association of class II pilE with strains that have particular epidemiological characteristics should become clearer.

A class II pilin gene was identified in one isolate from cc4, which was of particular interest as we also identified isolates in this clonal complex that possess a class I pilE gene (see Additional file 10). This was inconsistent with our previous analysis which indicated that isolates within clonal complexes were uniform with respect to pilin class (i.e. they all have either class I or class II pilE). cc4 meningococci are reported to be genetically homogeneous and were responsible for epidemics in the meningitis belt from the 1960s to the early 1990s [47]. The cc4 isolate with class II pilE was the oldest strain in the collection, isolated in 1937, while the two strains with class I pilE were from 1963 and 1983, suggesting that there may have been a shift from class II pilE to class I pilE in cc4. It is interesting to note that the cc4 complex has been replaced as the major cause of disease in the meningitis belt by $\mathrm{cc} 1$ or more recently cc5 meningococci, both of which have class II pilE [47].

Our analysis confirms that class II pilins in meningococci are antigenically stable. For example, we found that a serogroup W isolate (ID 20449, M11_240427, cc11) responsible for disease in the UK in 2011, has an identical pilE sequence to a serogroup B isolate (ID 349, 38VI, cc11), isolated from the USA in 1964. This contrasts with the finding that every class I pilin had a different sequence, even from strains with the same sequence type, isolated from the same country, during the same year. The extensive variation of class I pilE is consistent with the ability of single strains to generate pilin diversity through the recombination between pils cassettes and the pilE expression locus [48]. Indeed, for the majority of isolates with class I pilE the putative donor pilS cassettes could be identified. Although pils cassettes were found in all isolates which harboured a class II pilE gene, it was not possible to identify any class II pilE sequences that contained D-region information corresponding to pilS cassettes. Therefore, the WGS analysis provides further evidence for a lack of pilE/S recombination in class II pilin producing meningococci. A possible explanation for the low variability of the class II pilE genes is the absence of the G4 sequence that is required for pilin conversion. The current hypothesis is that the formation of a guanine quartet on the lagging strand stalls DNA replication, leading to a nick on the leading strand and initiating antigenic variation [49]. Therefore, in the absence of a functional G4 adjacent to pilE, these strains would lack the ability to initiate pilin conversion, although recent work suggests that additional factors may contribute to the lack of pilin variation [28]. In this respect, isolates expressing class II pilin provide a useful tool for systematically dissecting the molecular and genetic elements required for pilin variation.

These results also raise questions concerning the origin of the meningococcal class II pilE locus. The analysis of pilE genes in pathogenic and non-pathogenic Neisseria indicates that the $N$. meningitidis class II pilE is more closely related to pilE from non-pathogenic species than to the class I pilE from meningococci, in agreement with previous reports $[27,36]$. These observations are consistent with horizontal gene transfer among Neisseria species which has been observed by others $[38,50]$ and is likely, given that diverse species share a biological niche 
within the human host. Furthermore, we found one isolate of $N$. polysaccharea with the $h p$ gene adjacent to pilE. This gene, which encodes a putative transposase from Haemophilus influenzae, is consistently found next to the class II pilE gene in meningococci. Therefore this provides additional evidence in support of genetic exchange around the pilE locus among these species.

In addition to differences in pilE, meningococci producing class II pilin had fewer pilS cassettes (1-4 pilS) in the region between $l p x C$ and $f k b p$ than isolates with class I pilin (4-11 pilS). One possible explanation for this finding is that the $l p x C-f k b p$ region of existing class II strains represents a remnant of an ancestral class I pilE/ pils region, and that these class II isolates have at some point acquired a class II pilE gene and lost the class I expression locus and some pils cassettes. Interestingly, no genomes with both a class I and a class II pilE gene were found in this study. It is also noteworthy that recent genomic analysis indicates that pathogenic Neisseria evolved from a common ancestor shared with $N$. polysaccharea [50], which has a class II pilE gene. This could suggest a second possible scenario in which the class II pilE locus is ancestral in $N$. meningitidis and the class I pilS/pilE locus is a more recent trait which has arisen in specific lineages.

Tfp are surface structures which are exposed to the host environment in both colonisation and disease, and are thus presumed targets of the immune system. Consequently pilin variation has largely been assumed to provide meningococci with an effective mechanism of immune evasion. Indeed, anti-pilus antibodies can be found in patients infected with $N$. meningitidis [51] and gonococcal Tfp containing class I pilin are immunogenic in human volunteer studies [52,53] although there is no clear role for anti-PilE antibodies in protection against meningococcal infection [54]. Our finding that some clonal complexes express conserved class II pilin subunits is not consistent with host immunity-driven pilin variation in these strains, and is reminiscent of the lack of diversification of other surface proteins such as PorB, PorA, and FetA in some hyperinvasive strains $[55,56]$. However, we found some evidence of class II pilE gene exchange between unrelated isolates (i.e. different clonal complexes), indicating that recombination following uptake of exogenous DNA is a potential mechanism of generating class II pilin variation. A better understanding of the immune responses to class I and class II pilins should provide insight as to why distinct hyperinvasive lineages have evolved different potential for sequence variation of the Tfp major subunit.

\section{Conclusions}

In conclusion, we show that class II pilins can be defined by amino acid sequence and genomic context and are present in $N$. meningitidis strains which have persisted and spread globally over several decades and are responsible for meningococcal disease worldwide. Furthermore the class II pilE gene is present in non-pathogenic Neisseria, including $N$. polysaccharea and $N$. lactamica. Despite the presence of pils cassettes as potential donors of genetic variation in meningococci producing class II pilins, we found no evidence that class II pilE recombines with pils to generate pilin variation. Given the importance of $\mathrm{Tfp}$ in pathogen-host interaction, we propose that possession of class I or class II pilE is likely to impact bacterial behaviour, either through different capacity of strains to alter the sequence of the major pilus component or directly, through the inherent properties of the specific pilin subunits. For example, particular sequences of class I pilins affect key Tfp functions by influencing cell tropism or adhesion $[20,57,58]$. Additionally, specific amino acids in the D-region can affect pilus bundling [59] and this has recently been linked to serum resistance [23]. It is possible that class II pilins are highly conserved in certain lineages because they confer specific properties important for interactions within the host. Future work will be directed at understanding how these different pilin genes have evolved and their impact upon meningococcal biology.

\section{Methods}

\section{Genome database and collections}

The genome sequences of Neisseria strains used in this work are publically available online in the PubMLST Neisseria BIGSdb database (http://pubmlst.org/neisseria/). This database was developed by Keith Jolley and is sited at the University of Oxford [4]. The MRF-MGL (http://www. meningitis.org/research/genome) is an online open access library developed collaboratively by the Health Protection Agency, the Wellcome Trust Sanger Institute and the University of Oxford and is also hosted on the PubMLST website. The sequences analysed in this work were, for the most part, draft whole genome sequences and the majority were not fully assembled or closed genomes. The number and length of contigs for genomes can be found by using the "sequence bin" analysis tool on the PubMLST website. The 107 isolate MLST collection was accessed via the "Search Neisseria PubMLST database" web page using the "Publication" filter and selecting "Maiden et al. [2], Proc Nat Acad Sci USA 95:3140-5" from the drop down menu. The genomes of 514 meningococcal disease isolates from the epidemiological year 2010/11 in England, Wales and Northern Ireland were available in the MRF genome library (at time of writing). The genomes of 46 other Neisseria species were selected by excluding species names containing meningitidis and gonorrhoeae, and filtering for isolates with whole genome sequences in the drop down project menu. Each isolate has a unique ID number. Fully 
assembled (closed) genome sequences for reference strains MC58 (ID 240) [32], FAM18 (ID 698) [34], WUE2594 (ID 19260) [35], G2136 (ID638) [60], $\alpha 14$ (ID 30) [61], $\alpha 710$ (ID 19261) [62], M01-240355 (ID 19265) [60] , NZ-05/33 (ID 19263) [60], H44/76 (ID 237) [63], 8013 (ID 1038) [64] and N. lactamica 020-06 [65] are described in previous publications and are available on PubMLST.

\section{Identification of class I and class II pilE genes in meningococcal genomes}

Homologs of pilE were identified in the selected genomes using the BLASTn online analysis tool in PubMLST. For initial searches, the default parameters (word size 11 and 1 hit per isolate) were used. The query sequence consisted of either the full-length coding sequence of previously identified class I or class II pilE genes (e.g. MC58 [32] [Genbank: NC_003112] and FAM18 [26], [Genbank: NC_008767]) respectively or unpublished class II pilE sequences - R. Exley). The percentage identity between the nucleotide sequence of pilE from MC58 (class I) and FAM18 (class II) is $69 \%$ (Clustal W), and based on previous work [29] class II pilE genes are significantly shorter than class I pilE genes (approx. $450 \mathrm{bp}$ compared with $\geq 500 \mathrm{bp}$ ) therefore in most instances using a class I or a class II pilE coding sequence was sufficiently stringent to distinguish either a class I or a class II pilE homolog. However, to verify our findings, more comprehensive searches were also performed where the query sequence comprised nucleotides 1-180 of the pilE coding sequence from serogroup B strain MC58, which corresponds to the highly conserved $\mathrm{N}$ terminal alpha helical region of all meningococcal Type IVa pilins [66]. Hits with $>80 \%$ sequence identity over an alignment length of $\geq 50 \%$ of the query sequence were extracted with flanking sequence and manually inspected to identify pilE coding sequences and classify them as class I or class II based on the known characteristics of class I or class II pilins e.g: size, presence of the SM1 epitope EYYLN for class I pilins [25,26]. Using these search parameters we identified some genomes where pilE was not detected (Additional file 1). Given that the majority of the genome sequences are represented on contigs but are not closed, it was not possible to determine whether this was due to absence of pilE or insufficient contig assembly, therefore, these were excluded from further analysis. Additionally we excluded pilE sequences that were truncated by contig length as it was impossible to ascertain whether these were class I or class II pilE in the absence of full-length coding sequence. Finally, we also identified pilE genes that were either elongated, truncated by frameshift or had deletions compared to full-length pilE coding sequences. These sequences most likely resulted from gene conversion and would likely lead to production of a non-functional pilin subunit [67] and thus were also not included in further sequence analysis in this study. pilE was designated as NEIS0210 in PubMLST and each new nucleotide allele identified was assigned a unique number in the PubMLST definitions database. To identify whether any genomes contained more than one pilE gene, BLAST searches were performed using class I and class II pilE as query sequences, as well as selecting for up to 10 hits per isolate.

\section{Identification of pilE genes in non-pathogenic Neisseria} Homologs of pilE were identified in selected nonpathogenic Neisseria genomes using the BLASTn online analysis tool in PubMLST. For initial searches, the default parameters (word size 11, 1 hit per isolate and 5000 bp flanking length) were used. The query sequence consisted of the full-length pilE coding sequence of $N$. lactamica 020-06. Sequences were extracted and manually inspected to identify pilE. In species where two putative pilin genes were identified in tandem on the same contig, the gene with the higher homology to the N. lactamica 020-06 pilE was chosen for split decomposition network analysis.

\section{Identification of pilS cassettes in isolates containing class I pilE}

We analysed pilin loci in a subset of cc41/44, cc32, and cc269 isolates in order to determine the number and diversity of pilS cassettes residing in each pilE/S locus. We initially extracted the pilE/S locus from each strain by performing BLASTn searches in PubMLST using pilE as the query sequence; all search conditions were at default setting with the exception of a designated flanking length of 10,000 bp. The pilE-containing contigs were copied to serial cloner files and $f k b p$ and $l p x C$ genes were identified based on homology to previously annotated or published sequences. Sequences where contig length was insufficient to identify flanking genes were excluded from further analysis. We next identified individual pils cassettes within the $f k b p-l p x C$ regions of each isolate by utilizing the Align Two Sequences tool available at NCBI to interrogate the extracted $f k b p$ $l p x C$ region using the pilS1 gene from $N$. meningitidis strain M01-240149 as a query sequence in tBLASTn searches performed under default conditions. Each hit with $>60 \%$ sequence identity was inspected manually for features characteristic of known neisserial pils cassettes, which have been defined as partial genes with homology to pilin-encoding sequences $[31,68]$. We delineated the $5^{\prime}$ and 3 ' boundaries of each pils cassette based on this criterion, although in some cases open reading frames extended beyond the region of pilin homology. 


\section{Identification of pilS cassettes in class II pilE-containing isolates}

The genome sequence of each isolate with class II pilE was first examined for pils-containing regions using the BLASTn online analysis tool on PubMLST using pilS1 and pilS2 genes from $N$. meningitidis strain FAM18 as query sequences under default conditions. Sequence regions containing hits were extracted with up to $5000 \mathrm{bp}$ of flanking sequence as allowable by contig length. We examined each extracted pilS-containing region for $f k b p$ homology, lpxC homology, and number and diversity of pilS cassettes as indicated above. Comparisons of pilE and pils sequences from each strain were also carried out as indicated above.

Identification of Sma/Cla and Guanine quartet sequences The sequence of the $66 \mathrm{bp} \mathrm{Sma/Cla} \mathrm{repeats} \mathrm{from} N$. gonorrhoeae and $N$. meningitidis strain MC58 reported in $[10,37]$ were used as a query sequences to scan pilE loci for identical sequences. Where identical sequences were not identified, we manually inspected loci for highly similar sequences. Of note, the SmaI (CCCGGG) and ClaI (ATCGAT) recognition sites were not present in the query sequence and are not consistently present flanking this repeat in all genomes. However, for consistency we have kept the name Sma/Cla repeat. The sequences reported in Cahoon and Seifert [12] were used to identify putative G4 based on sequence homology.

\section{Sequence analysis, annotation and illustration}

The online sequence analysis tool NRDB (http://pubmlst. org/analysis/) was used to identify unique alleles and EMBOSS transeq (http://www.ebi.ac.uk/Tools/st/emboss_transeq/) was used to translate multiple coding sequences for protein sequence analysis. Nucleotide and amino acid alignments, including comparisons of pilE and pils loci from each isolate, were carried out using the online analysis tool MUSCLE available at EBI [69] or Clustal W (http://www. ebi.ac.uk/Tools/msa/clustalw2/) [70]. Alignment was displayed using the BoxShade 3.31 program on the Mobyle platform [71]. Genome regions were annotated by performing BLAST analysis with pilE (class I or class II), or alternatively $f k p b$ or $l p x C$ as the query sequence and downloading flanking regions (usually up to 10,000 bp) from the pubMLST website. Coding sequences on the contigs were identified based on homology to previously published and/or annotated sequences [32-34,64,72,73]. Gene diagrams of regions between $f k b p$ and $l p x C$ and $k a t A$ and $p r l C$ by were manually constructed and drawn to scale using Microsoft PowerPoint.

\section{Phylogenetic network analysis}

The SplitsTree decomposition network of pilE sequences was obtained by performing sequence alignment in MEGA
5.0 [74] and network analysis using Splitstree4 [75]. Fulllength nucleotide sequences of pilE homologues from non-pathogenic Neisseria species were compared with pilE genes from $14 N$. meningitidis isolates which are reference isolates with previously published and annotated whole genomes (listed in Additional files 2, 3 and 4), and/ or represent lineages with class II pilE identified in this work (i.e. cc1, cc4 and cc174).

\section{Western blot analysis of SM1 cross reactivity of class II pilins}

N. meningitidis strains FAM18 (ID 698), 8013 (ID 1038) and 92001 (ID 597) and isogenic mutants in which the pilE coding sequence is replaced by a kanamycin resistance cassette were grown overnight on Brain Heart Infusion (BHI) agar $\left(1.5 \% \mathrm{wt} / \mathrm{vol}\right.$, Oxoid) at $37^{\circ} \mathrm{C}$ in the presence of $5 \% \mathrm{CO}_{2}$. Bacteria $\left(1 \times 10^{9} \mathrm{CFU}\right)$ were resuspended in $50 \mu \mathrm{l}$ of sterile water and an equal volume of $2 \times$ SDS-PAGE lysis buffer was added before boiling for $10 \mathrm{mi}$ nutes. $10 \mu \mathrm{l}$ of each whole cell extract was separated on $12 \%$ polyacrylamide gels and transferred to PVDF membrane for Western blotting. SM1 antibody (a kind gift from M. Virji) was used at a final concentration of 1:10,000 and antibody binding was detected by incubation of membranes for one hour with a goat anti-mouse HRP conjugate (DAKO) at 1:10,000. An anti-peptide antibody generated by immunisation of rabbits with peptide CGQKSAVTEYYLNHGE (Eurogentec) was used to detect both class I and class II pilin expression at a final concentration of 1:5000, followed by an anti-rabbit HRP conjugate (1:10,000; Santa Cruz). Cross reaction was detected using ECL detection reagent (GE Healthcare).

\section{Availability of supporting data}

The draft or complete genomes available in this article are publically available online as described. Additional files with supporting results are included with this article.

\section{Additional files}

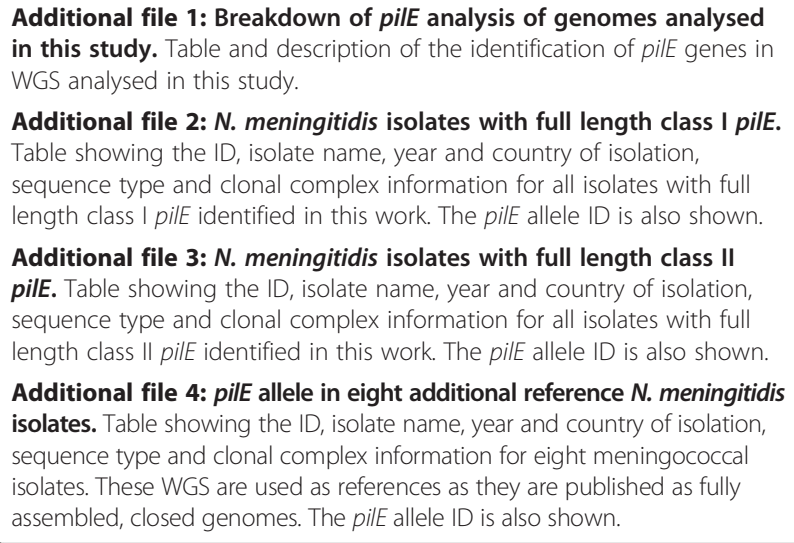

Additional file 1: Breakdown of pilE analysis of genomes analysed in this study. Table and description of the identification of pilE genes in WGS analysed in this study.

Additional file 2: $\mathbf{N}$. meningitidis isolates with full length class I pilE. Table showing the ID, isolate name, year and country of isolation, sequence type and clonal complex information for all isolates with full length class I pilE identified in this work. The pilE allele ID is also shown.

Additional file 3: $N$. meningitidis isolates with full length class II pilE. Table showing the ID, isolate name, year and country of isolation, sequence type and clonal complex information for all isolates with full length class II pilE identified in this work. The pilE allele ID is also shown.

Additional file 4: pilE allele in eight additional reference $N$. meningitidis isolates. Table showing the ID, isolate name, year and country of isolation, sequence type and clonal complex information for eight meningococcal isolates. These WGS are used as references as they are published as fully assembled, closed genomes. The pilE allele ID is also shown. 
Additional file 5: Schematic diagrams of class I pilE regions. Schematic representation of class I pilE/S regions and katA-prlC regions from isolates belonging to clonal complex (cc) 41/44 and cc262.

Additional file 6: Schematic diagrams of class II pilE regions. Schematic representation of class II pilE regions and pilS regions from isolates belonging to $\mathrm{CC} 1, \mathrm{cc} 8, \mathrm{cc} 174$ and $\mathrm{Cc} 11$.

Additional file 7: Sequences of putative G4 and Sma Cla sequences annotated in this study. Table of the G4 and Sma/Cla sequences identified in the pilE/pilS regions of WGS annotated in this study.

Additional file 8: Non-pathogenic Neisseria isolates examined in this study. Table describing the origin and details of the non-pathogenic Neisseria WGS analysed in this work. The number of putative pilE genes identified is also indicated.

Additional file 9: Schematic diagrams of pilE regions of $N$. lactamica and $N$. polysaccharea. Schematic representation of pilE regions in $N$. lactamica and N. polysaccharea WGS analysed in this study.

Additional file 10: Schematic diagram of pilE regions in cc4 isolates. Schematic representation showing the genetic context of the class I or class II pilE genes identified in cc4 meningococcal isolates.

\section{Competing interests}

The authors declare that they have no competing interests.

\section{Authors' contributions}

$\mathrm{RE}$ and $\mathrm{CT}$ conceived the idea for this article. MW and RE analysed the pilE sequences, $\mathrm{CH}$ and EA analysed pilS loci, MW and JB analysed pilE sequences from non-pathogenic Neisseria, KJ and MM devised the PubMLST database and contributed to all aspects of data extraction and analysis. RE, EA, CT, MW wrote the manuscript. All authors read and approved the final submission.

\section{Acknowledgements}

RE, MW and CT are funded by the Wellcome Trust (WT093470AIA). MCJM is a Wellcome Trust Senior Research Fellow in Basic Biomedical Sciences. The Meningitis Research Foundation Meningococcus Genome Library was funded by the Meningitis Research Foundation. The Neisseria Multi Locus Sequence Typing website was funded by the Wellcome Trust and European Union. We thank Lydia Stinar and Stephen Rostad for assistance with the pils analysis.

\section{Author details}

${ }^{1}$ Sir William Dunn School of Pathology, University of Oxford, Oxford OX1 3RE, UK. ${ }^{2}$ Department of Biology, Concordia College, Moorhead, MN, USA.

${ }^{3}$ Department of Zoology, University of Oxford, Oxford OX1 3PS, UK.

Received: 29 November 2013 Accepted: 25 March 2014

Published: 1 April 2014

\section{References}

1. Pace D, Pollard AJ: Meningococcal disease: clinical presentation and sequelae. Vaccine 2012, 30(Suppl 2):B3-B9.

2. Maiden MC, Bygraves JA, Feil E, Morelli G, Russell JE, Urwin R, Zhang Q Zhou J, Zurth K, Caugant DA, Feavers IM, Achtman M, Spratt BG: Multilocus sequence typing: a portable approach to the identification of clones within populations of pathogenic microorganisms. Proc Natl Acad Sci U S A 1998, 95(6):3140-3145.

3. Caugant DA, Maiden MC: Meningococcal carriage and disease-population biology and evolution. Vaccine 2009, 27(Suppl 2):B64-B70.

4. Jolley KA, Maiden MC: BIGSdb: scalable analysis of bacterial genome variation at the population level. BMC Bioinformatics 2010, 11:595.

5. Carbonnelle E, Hill DJ, Morand P, Griffiths NJ, Bourdoulous S, Murillo I, Nassif X, Virji M: Meningococcal interactions with the host. Vaccine 2009, 27(Suppl 2):B78-B89.

6. Trivedi K, Tang CM, Exley RM: Mechanisms of meningococcal colonisation. Trends Microbiol 2011, 19(9):456-463.

7. Seifert HS, Ajioka RS, Paruchuri D, Heffron F, So M: Shuttle mutagenesis of Neisseria gonorrhoeae: pilin null mutations lower DNA transformation competence. J Bacteriol 1990, 172(1):40-46.
8. Hagblom P, Segal E, Billyard E, So M: Intragenic recombination leads to pilus antigenic variation in Neisseria gonorrhoeae. Nature 1985, 315(6015):156-158.

9. Segal E, Billyard E, So M, Storzbach S, Meyer TF: Role of chromosomal rearrangement in N. gonorrhoeae pilus phase variation. Cell 1985, 40(2):293-300.

10. Wainwright LA, Pritchard KH, Seifert HS: A conserved DNA sequence is required for efficient gonococcal pilin antigenic variation. Mol Microbiol 1994, 13(1):75-87.

11. Kuryavyi V, Cahoon LA, Seifert HS, Patel DJ: RecA-binding pilE G4 sequence essential for pilin antigenic variation forms monomeric and $5{ }^{\prime}$ end-stacked dimeric parallel G-quadruplexes. Structure 2012, 20(12):2090-2102.

12. Cahoon LA, Seifert HS: An alternative DNA structure is necessary for pilin antigenic variation in Neisseria gonorrhoeae. Science 2009, 325(5941):764-767.

13. Cahoon LA, Seifert HS: Transcription of a cis-acting, noncoding, small RNA is required for pilin antigenic variation in Neisseria gonorrhoeae. PLoS Pathog 2013, 9(1):e1003074.

14. Forest KT, Bernstein SL, Getzoff ED, So M, Tribbick G, Geysen HM, Deal CD, Tainer JA: Assembly and antigenicity of the Neisseria gonorrhoeae pilus mapped with antibodies. Infect Immun 1996, 64(2):644-652.

15. Criss AK, Kline KA, Seifert HS: The frequency and rate of pilin antigenic variation in Neisseria gonorrhoeae. Mol Microbiol 2005, 58(2):510-519.

16. Hamrick TS, Dempsey JA, Cohen MS, Cannon JG: Antigenic variation of gonococcal pilin expression in vivo: analysis of the strain FA1090 pilin repertoire and identification of the pilS gene copies recombining with pilE during experimental human infection. Microbiology 2001, 147(Pt 4):839-849.

17. Helm RA, Seifert HS: The frequency and rate of pilin antigenic variation of Neisseria meningitidis. I Bacteriol 2010. In press.

18. Omer H, Rose G, Jolley KA, Frapy E, Zahar JR, Maiden MC, Bentley SD, Tinsley CR, Nassif X, Bille E: Genotypic and phenotypic modifications of Neisseria meningitidis after an accidental human passage. PloS One 2011, 6(2):e17145.

19. Tinsley CR, Heckels JE: Variation in the expression of pili and outer membrane protein by Neisseria meningitidis during the course of meningococcal infection. J Gen Microbiol 1986, 132(9):2483-2490.

20. Virji M, Alexandrescu C, Ferguson DJ, Saunders JR, Moxon ER: Variations in the expression of pili: the effect on adherence of Neisseria meningitidis to human epithelial and endothelial cells. Mol Microbiol 1992, 6(10):1271-1279.

21. Nassif $X$, Lowy J, Stenberg P, O'Gaora P, Ganji A, So M: Antigenic variation of pilin regulates adhesion of Neisseria meningitidis to human epithelial cells. Mol Microbiol 1993, 8(4):719-725.

22. Virji M, Saunders JR, Sims G, Makepeace K, Maskell D, Ferguson DJ: Pilus-facilitated adherence of Neisseria meningitidis to human epithelial and endothelial cells: modulation of adherence phenotype occurs concurrently with changes in primary amino acid sequence and the glycosylation status of pilin. Mol Microbiol 1993, 10(5):1013-1028.

23. Hubert K, Pawlik MC, Claus H, Jarva H, Meri S, Vogel U: Opc expression, LPS immunotype switch and pilin conversion contribute to serum resistance of unencapsulated meningococci. PloS One 2012, 7(9):e45132.

24. Virji M, Heckels JE: Antigenic cross-reactivity of Neisseria pili: investigations with type- and species-specific monoclonal antibodies. J Gen Microbiol 1983, 129(9):2761-2768.

25. Virji M, Heckels JE, Potts WJ, Hart CA, Saunders JR: Identification of epitopes recognized by monoclonal antibodies SM1 and SM2 which react with all pili of Neisseria gonorrhoeae but which differentiate between two structural classes of pili expressed by Neisseria meningitidis and the distribution of their encoding sequences in the genomes of Neisseria spp. J Gen Microbiol 1989, 135(12):3239-3251.

26. Aho EL, Botten JW, Hall RJ, Larson MK, Ness JK: Characterization of a class II pilin expression locus from Neisseria meningitidis: evidence for increased diversity among pilin genes in pathogenic Neisseria species. Infect Immun 1997, 65(7):2613-2620.

27. Aho EL, Keating AM, McGillivray SM: A comparative analysis of pilin genes from pathogenic and nonpathogenic Neisseria species. Microb Pathog 2000, 28(2):81-88.

28. Davies JK, Harrison PF, Lin YH, Bartley S, Khoo CA, Seemann T, Ryan CS, Kahler CM, Hill SA: The use of high-throughput DNA sequencing in the 
investigation of antigenic variation: application to Neisseria species. Plos One 2014, 9(1):e86704.

29. Cehovin A, Winterbotham M, Lucidarme J, Borrow R, Tang CM, Exley RM, Pelicic V: Sequence conservation of pilus subunits in Neisseria meningitidis. Vaccine 2010, 28(30):4817-4826.

30. Sun X, Zhou H, Xu L, Yang H, Gao Y, Zhu B, Shao Z: Prevalence and genetic diversity of two adhesion-related genes, pilE and nadA, in Neisseria meningitidis in China. Epidemiol Infect 2013, 141(10):2163-2172.

31. Haas R, Meyer TF: The repertoire of silent pilus genes in Neisseria gonorrhoeae: evidence for gene conversion. Cell 1986, 44(1):107-115.

32. Tettelin $H$, Saunders NJ, Heidelberg J, Jeffries AC, Nelson KE, Eisen JA, Ketchum KA, Hood DW, Peden JF, Dodson RJ, Nelson WC, Gwinn ML, DeBoy R, Peterson JD, Hickey EK, Haft DH, Salzberg SL, White O, Fleischmann RD, Dougherty BA, Mason T, Ciecko A, Parksey DS, Blair E, Cittone $\mathrm{H}$, Clark EB, Cotton MD, Utterback TR, Khouri H, Qin H, et al: Complete genome sequence of Neisseria meningitidis serogroup B strain MC58. Science 2000, 287(5459):1809-1815.

33. Parkhill J, Achtman M, James KD, Bentley SD, Churcher C, Klee SR, Morelli G, Basham D, Brown D, Chillingworth T, Davies RM, Davis P, Devlin K, Feltwell T, Hamlin N, Holroyd S, Jagels K, Leather S, Moule S, Mungall K, Quail MA, Rajandream MA, Rutherford KM, Simmonds M, Skelton J, Whitehead S, Spratt BG, Barrell BG: Complete DNA sequence of a serogroup A strain of Neisseria meningitidis Z2491. Nature 2000, 404(6777):502-506.

34. Bentley SD, Vernikos GS, Snyder LA, Churcher C, Arrowsmith C, Chillingworth T, Cronin A, Davis PH, Holroyd NE, Jagels K, Maddison M, Moule S, Rabbinowitsch E, Sharp S, Unwin L, Whitehead S, Quail MA, Achtman M, Barrell B, Saunders NJ, Parkhill J: Meningococcal genetic variation mechanisms viewed through comparative analysis of serogroup C strain FAM18. PLoS Genet 2007, 3(2):e23.

35. Schoen C, Weber-Lehmann J, Blom J, Joseph B, Goesmann A, Strittmatter A, Frosch M: Whole-genome sequence of the transformable Neisseria meningitidis serogroup A strain WUE2594. J Bacteriol 2011, 193(8):2064-2065.

36. Aho EL, Urwin R, Batcheller AE, Holmgren AM, Havig K, Kulakoski AM, Vomhof EE, Longfors NS, Erickson CB, Anderson ZK, Dawlaty JM, Mueller JJ: Neisserial pilin genes display extensive interspecies diversity. FEMS Microbiol Lett 2005, 249(2):327-334.

37. Wainwright LA, Frangipane $\mathrm{J}$, Seifert HS: Analysis of protein binding to the Sma/Cla DNA repeat in pathogenic Neisseriae. Nucleic Acids Res 1997, 25(7):1362-1368

38. Marri PR, Paniscus M, Weyand NJ, Rendon MA, Calton CM, Hernandez DR, Higashi DL, Sodergren E, Weinstock GM, Rounsley SD, So M: Genome sequencing reveals widespread virulence gene exchange among human Neisseria species. PloS One 2010, 5(7):e11835.

39. Kahler CM, Martin LE, Tzeng YL, Miller YK, Sharkey K, Stephens DS, Davies JK: Polymorphisms in pilin glycosylation Locus of Neisseria meningitidis expressing class II pili. Infect Immun 2001, 69(6):3597-3604.

40. Caugant DA: Genetics and evolution of Neisseria meningitidis: importance for the epidemiology of meningococcal disease. Infect Genet Evol 2008, 8(5):558-565.

41. Chang Q, Tzeng YL, Stephens DS: Meningococcal disease: changes in epidemiology and prevention. Clin Epidemio/ 2012, 4:237-245.

42. Nicolas P, Norheim G, Garnotel E, Djibo S, Caugant DA: Molecular epidemiology of neisseria meningitidis isolated in the African Meningitis Belt between 1988 and 2003 shows dominance of sequence type 5 (ST-5) and ST-11 complexes. J Clin Microbiol 2005, 43(10):5129-5135.

43. Nicolas P, Decousset L, Riglet V, Castelli P, Stor R, Blanchet G: Clonal expansion of sequence type (ST-)5 and emergence of ST-7 in serogroup A meningococci, Africa. Emerg Infect Dis 2001, 7(5):849-854.

44. Teyssou R, Muros-Le Rouzic E: Meningitis epidemics in Africa: a brief overview. Vaccine 2007, 25(Suppl 1):A3-A7.

45. Ibarz-Pavon AB, Maclennan J, Andrews NJ, Gray SJ, Urwin R, Clarke SC, Walker AM, Evans MR, Kroll JS, Neal KR, Ala'aldeen D, Crook DW, Cann K, Harrison S, Cunningham R, Baxter D, Kaczmarski E, McCarthy ND, Jolley KA, Cameron JC, Stuart JM, Maiden MC: Changes in serogroup and genotype prevalence among carried meningococci in the United Kingdom during vaccine implementation. J Infect Dis 2011, 204(7):1046-1053.

46. Ladhani SN, Lucidarme J, Newbold LS, Gray SJ, Carr AD, Findlow J, Ramsay ME, Kaczmarski EB, Borrow R: Invasive meningococcal capsular group $\mathrm{Y}$ disease, England and Wales, 2007-2009. Emerg Infect Dis 2012, 18(1):63-70.

47. Leimkugel J, Racloz V, Jacintho da Silva L, Pluschke G: Global review of meningococcal disease. A shifting etiology. J Bacterio/ Res 2009, 1(1):006-018.
48. Cahoon LA, Seifert HS: Focusing homologous recombination: pilin antigenic variation in the pathogenic Neisseria. Mol Microbiol 2011, 81(5):1136-1143.

49. Cahoon LA, Manthei KA, Rotman E, Keck JL, Seifert HS: Neisseria gonorrhoeae RecQ helicase HRDC domains are essential for efficient binding and unwinding of the pilE guanine quartet structure required for pilin antigenic variation. J Bacteriol 2013, 195(10):2255-2261.

50. Bennett JS, Jolley KA, Earle SG, Corton C, Bentley SD, Parkhill J, Maiden MC: A genomic approach to bacterial taxonomy: an examination and proposed reclassification of species within the genus Neisseria. Microbiology 2012, 158(Pt 6):1570-1580.

51. Poolman JT, Hopman CT, Zanen HC: Immunogenicity of meningococcal antigens as detected in patient sera. Infect Immun 1983, 40(1):398-406.

52. Tramont EC, Sadoff JC, Boslego JW, Ciak J, McChesney D, Brinton CC, Wood S, Takafuji E: Gonococcal pilus vaccine. Studies of antigenicity and inhibition of attachment. J Clin Invest 1981, 68(4):881-888.

53. Cohen MS, Cannon JG: Human experimentation with Neisseria gonorrhoeae: progress and goals. J Infect Dis 1999, 179(Suppl 2):S375-S379.

54. Cehovin A, Kroll JS, Pelicic V: Testing the vaccine potential of PilV, PilX and ComP, minor subunits of Neisseria meningitidis type IV pili. Vaccine 2011, 29(40):6858-6865.

55. Huber CA, Pfluger V, Hamid AW, Forgor AA, Hodgson A, Sie A, Junghanss T, Pluschke G: Lack of antigenic diversification of major outer membrane proteins during clonal waves of Neisseria meningitidis serogroup A colonization and disease. Pathog Dis 2013, 67(1):4-10.

56. Watkins ER, Maiden MC: Persistence of hyperinvasive meningococcal strain types during global spread as recorded in the PubMLST database. Plos One 2012, 7(9):e45349.

57. Jonsson AB, Ilver D, Falk P, Pepose J, Normark S: Sequence changes in the pilus subunit lead to tropism variation of Neisseria gonorrhoeae to human tissue. Mol Microbiol 1994, 13(3):403-416.

58. Hardy SJ, Christodoulides M, Weller RO, Heckels JE: Interactions of Neisseria meningitidis with cells of the human meninges. Mol Microbiol 2000, 36(4):817-829.

59. Marceau M, Beretti $\lrcorner$, Nassif X: High adhesiveness of encapsulated Neisseria meningitidis to epithelial cells is associated with the formation of bundles of pili. Mol Microbiol 1995, 17(5):855-863.

60. Budroni S, Siena E, Dunning Hotopp JC, Seib KL, Serruto D, Nofroni C, Comanducci M, Riley DR, Daugherty SC, Angiuoli SV, Covacci A, Pizza M, Rappuoli R, Moxon ER, Tettelin H, Medini D: Neisseria meningitidis is structured in clades associated with restriction modification systems that modulate homologous recombination. Proc Natl Acad Sci U S A 2011, 108(11):4494-4499.

61. Schoen C, Blom J, Claus H, Schramm-Gluck A, Brandt P, Muller T, Goesmann A, Joseph B, Konietzny S, Kurzai O, Schmitt C, Friedrich T, Linke B, Vogel U, Frosch M: Whole-genome comparison of disease and carriage strains provides insights into virulence evolution in Neisseria meningitidis. Proc Natl Acad Sci U S A 2008, 105(9):3473-3478.

62. Joseph B, Schneiker-Bekel S, Schramm-Gluck A, Blom J, Claus H, Linke B, Schwarz RF, Becker A, Goesmann A, Frosch M, Schoen C: Comparative genome biology of a serogroup B carriage and disease strain supports a polygenic nature of meningococcal virulence. J Bacterio/ 2010, 192(20):5363-5377.

63. Piet JR, Huis in 't Veld RA, van Schaik BD, van Kampen AH, Baas F, van de Beek D, Pannekoek Y, van der Ende A: Genome sequence of Neisseria meningitidis serogroup B strain H44/76. J Bacteriol 2011, 193(9):2371-2372.

64. Rusniok C, Vallenet D, Floquet $S$, Ewles H, Mouze-Soulama C, Brown D, Lajus A, Buchrieser C, Medigue C, Glaser P, Pelicic V: NeMeSys: a biological resource for narrowing the gap between sequence and function in the human pathogen Neisseria meningitidis. Genome Biol 2009, 10(10):R110.

65. Bennett JS, Bentley SD, Vernikos GS, Quail MA, Cherevach I, White B, Parkhill J, Maiden MC: Independent evolution of the core and accessory gene sets in the genus Neisseria: insights gained from the genome of Neisseria lactamica isolate 020-06. BMC Genomics 2010, 11:652.

66. Craig L, Pique ME, Tainer JA: Type IV pilus structure and bacterial pathogenicity. Nat Rev Microbiol 2004, 2(5):363-378

67. Manning PA, Kaufmann A, Roll U, Pohlner J, Meyer TF, Haas R: L-pilin variants of Neisseria gonorrhoeae MS11. Mol Microbiol 1991, 5(4):917-926.

68. Perry AC, Nicolson IJ, Saunders JR: Neisseria meningitidis C114 contains silent, truncated pilin genes that are homologous to Neisseria gonorrhoeae pil sequences. J Bacteriol 1988, 170(4):1691-1697. 
69. Edgar RC: MUSCLE: multiple sequence alignment with high accuracy and high throughput. Nucleic Acids Res 2004, 32(5):1792-1797.

70. Larkin MA, Blackshields G, Brown NP, Chenna R, McGettigan PA, McWilliam H, Valentin F, Wallace IM, Wilm A, Lopez R, Thompson JD, Gibson TJ, Higgins DG: Clustal W and Clustal X version 2.0. Bioinformatics 2007, 23(21):2947-2948.

71. Neron B, Menager H, Maufrais C, Joly N, Maupetit J, Letort S, Carrere S, Tuffery $P$, Letondal C: Mobyle: a new full web bioinformatics framework. Bioinformatics 2009, 25(22):3005-3011.

72. Johnson SR, Steiner BM, Perkins GH: Cloning and characterization of the catalase gene of Neisseria gonorrhoeae: use of the gonococcus as a host organism for recombinant DNA. Infect Immun 1996, 64(7):2627-2634.

73. Sampson BA, Gotschlich EC: Neisseria meningitidis encodes an FK506-inhibitable rotamase. Proc Natl Acad Sci U S A 1992, 89(4):1164-1168.

74. Tamura K, Peterson D, Peterson N, Stecher G, Nei M, Kumar S: MEGA5: molecular evolutionary genetics analysis using maximum likelihood, evolutionary distance, and maximum parsimony methods. Mol Biol Evol 2011, 28(10):2731-2739.

75. Huson DH: SplitsTree: analyzing and visualizing evolutionary data. Bioinformatics 1998, 14(1):68-73.

doi:10.1186/1471-2164-15-253

Cite this article as: Wörmann et al:: Sequence, distribution and chromosomal context of class I and class II pilin genes of Neisseria meningitidis identified in whole genome sequences. BMC Genomics 2014 15:253.

\section{Submit your next manuscript to BioMed Central and take full advantage of:}

- Convenient online submission

- Thorough peer review

- No space constraints or color figure charges

- Immediate publication on acceptance

- Inclusion in PubMed, CAS, Scopus and Google Scholar

- Research which is freely available for redistribution 